\title{
Avaliação nutricional de gestantes sob acompanhamento em serviços de pré-natal distintos: a região metropolitana e o ambiente rural
}

\section{Nutritional Status of Pregnant Women under Monitoring in Pre Distinct Prenatal Services: The Metropolitan Area and the Rural Environment}

\author{
Caroline San Severino Teixeira ${ }^{1}$ Antônio Carlos Vieira Cabral ${ }^{2}$ \\ 1 Programa de Pós-Graduação em Saúde da Mulher (Mestrado) da \\ Universidade Federal de Minas Gerais, Belo Horizonte, MG, Brasil \\ 2 Faculdade de Medicina da Universidade Federal de Minas Gerais, Belo \\ Horizonte, MG, Brasil

\begin{abstract}
Address for correspondence Caroline San Severino Teixeira, Nutricionista, Rua Raimundo Ludgero Alves, 05. Centro, Paula Cândido, MG, Brasil CEP: 36544-000
\end{abstract} \\ (e-mail: carolinesanseverino@yahoo.com.br).
}

Rev Bras Ginec Obst 2016;38:27-34.

\section{Resumo}

Palavras-Chave

- gestação

- hábitos alimentares

- nutrição pré-natal

- estado nutricional

- ganho de peso
Objetivo Verificar diferenças em alguns aspectos nutricionais de gestantes acompanhadas em serviço de atenção pré-natal em uma cidade do interior e na região metropolitana.

Métodos Foram avaliadas gestantes em atendimento pré-natal na cidade de Belo Horizonte (BH), região metropolitana, e Paula Cândido (PC), interior de MG. Aplicou-se um Questionário de Frequência Alimentar (QFA) contendo informações socioeconômicas e sobre o hábito alimentar, além disso, foram aferidos peso e altura no momento do atendimento e questionado o peso pré-gestacional, para posterior cálculo do IMC (índice de massa corpórea). A análise dos dados foi dividida por região e trimestre gestacional, utilizando o software SPSS versão 15.0, teste t para comparação de médias e qui-quadrado de independência, com $5 \%$ de significância.

Resultados Foram incluídas 240 gestantes, sendo 90 do interior e 150 da região metropolitana. Destas, a maioria são casadas ( $\mathrm{BH}=56,6 \%$; $\mathrm{PC}=46,6 \%)$, não trabaIham fora de casa ( $\mathrm{BH}=54,6 \% ; \mathrm{PC}=84,4 \%$ ), predominantemente se alimentam 3 a 4 vezes ao dia no $1^{\circ}$ e $2^{\circ}$ trimestre $(\mathrm{BH}=54,0$ e $46,0 \%$; $\mathrm{PC}=66,7$ e $63,3 \%$, respectivamente) e fazem 5 a 6 refeições ao dia no $3^{\circ}$ trimestre em $\mathrm{BH}(44 \%)$. Houve ganho de peso significativo somente no $1^{\circ}$ trimestre (BH: 58,0\%; PC: $53,33 \%$ ). Ganho de peso versus hábito alimentar foi significativo para as variáveis "almoça ou janta fora de casa," no $1^{\circ}$ trimestre $\mathrm{BH}(p=0,006)$; "quantas vezes consome leite," no $1^{\circ}$ trimestre PC $(p=0,03)$; "quantas vezes consome fastfood," no $3^{\circ}$ trimestre $\mathrm{BH}(p=0,009)$.

Conclusões As gestantes em ambas regiões se alimentam de forma adequada, apesar da prevalência de sobrepeso pré-gestacional em $\mathrm{BH}$ e baixo nível de escolaridade e received

May 14, 2015

accepted

October 19, 2015
DOI http://dx.doi.org/ 10.1055/s-0035-1570111. ISSN 0100-7203.
Copyright $(\underset{0}{ } 2016$ by Thieme Publicações License terms

Ltda, Rio de Janeiro, Brazil
(요 (1) $\Theta \circledast$ 


\begin{abstract}
Keywords

- pregnancy

- eating habits

- prenatal nutrition

- nutritional status

- weight gain

Objective To determine differences in some nutritional aspects of pregnant women assisted at prenatal care services in a country town and in a metropolitan area.

Methods Pregnant women received prenatal care in the city of Belo Horizonte (BH), metropolitan area, and Paula Cândido (PC), a country town. A Food Frequency Questionnaire (FFQ) containing socioeconomic information and information about eating habits was applied. In addition,weight and height were measured on the occasion of the visits and the women were ask to give their prepregnancy weight for subsequent BMI calculation. Data were analyzed according to region and trimester of pregnancy using the SPSS software version 15.0, the $t$-test to compare averages and the chi-square test of independence, with the level of significance set at $5 \%$.

Results 240 pregnant women were included, 90 from the country town and 150 from the metropolitan area. Of these, most were married $(\mathrm{BH}=56.6 \%$; $\mathrm{PC}=46.6 \%)$ and did not work outside the home $(\mathrm{BH}=54.0 \% ; \mathrm{PC}=84.4 \%)$. They predominantly had 3-4 meals/ day during the $1^{\text {st }}$ and $2^{\text {nd }}$ quarters $(\mathrm{BH}=54.0$ and $46 \% ; \mathrm{PC}=66.7$ and $63.3 \%$, respectively) and had 5-6 meals/day during Q3 in $\mathrm{BH}(44 \%)$. There was significant weight gain only in the 1 st quarter (BH: $58,0 \%$; PC: $53.3 \%$ ). Weight gain versus eating habits was significant for the variables "lunch or dinner away from home" for the $1^{\text {st }}$ quarter in $\mathrm{BH}(p=0.006)$, "How many times they consume milk" in the $1^{\text {st }}$ quarter in $\mathrm{PC}(p=0.03)$, and "How many times they consume junk food" in the $3^{\text {rd }}$ quarter in $\mathrm{BH}$ $(p=0.009)$.

Conclusions Pregnant woman showed proper eating habits in both regions despite the prevalence of pregestational overweight in $\mathrm{BH}$ and a low level of education and income, especially in the country town, an indicator that may be unfavorable for the nutrition of pregnant women during this period. Studies of association between eating habits and newborn health will provide more information about nutrition during pregnancy.
\end{abstract}

renda, principalmente no interior, indicador que pode ser pouco favorável à nutrição das gestantes neste período. Estudos de associação entre hábito alimentar e saúde do recém-nascido irão contribuir para maiores informações sobre a nutrição no período gestacional.

\section{Introdução}

A gestação é um período que impõe necessidades nutricionais aumentadas, e a adequada nutrição é primordial para a saúde da mãe e do feto. Gestantes devem consumir alimentos em variedade e quantidade específicas, considerando as recomendações dos guias alimentares e as práticas alimentares culturais, para atingir as necessidades energéticas e nutricionais, e as recomendações de ganho de peso. ${ }^{1}$

As gestantes são suscetíveis à inadequação nutricional, pelo aumento da demanda de energia, macro e micronutrientes, que ocorrem durante a gravidez. A qualidade da alimentação e o estado nutricional da mulher, antes e durante a gravidez, afetam o crescimento e o desenvolvimento fetal, bem como a evolução da gestação. ${ }^{2}$

A inadequação do ganho de peso durante a gestação tem sido apontada como fator de risco tanto para a mãe quanto para o concepto. ${ }^{3} \mathrm{O}$ ganho de peso aquém do recomendado pode acarretar restrição de crescimento intrauterino, parto prematuro, baixo peso ao nascer e aumento das taxas de morbimortalidade perinatal. ${ }^{4,5} \mathrm{O}$ ganho excessivo está asso- ciado, no feto, a, macrossomia, desproporção céfalo-pélvica, asfixia e na mãe, diabetes mellitus gestacional, hipertensão arterial, pré-eclâmpsia, eclampsia, maior retenção de peso pós-parto e aumento do risco de obesidade futura. ${ }^{6,7}$

Neste sentido, é fundamental dispor de instrumentos capazes de avaliar a ingestão alimentar materna de forma a identificar, com eficácia e precisão, associações diretas entre a alimentação e a saúde da mãe e do feto. Na literatura atual sobre as relações entre a alimentação, saúde e prevenção de doenças não transmissíveis destaca-se, precisamente, o interesse exponencial em examinar a alimentação humana numa perspectiva multidimensional, por oposição à visão direcionada para apenas um nutriente, alimento, ou mesmo, grupo de alimentos. ${ }^{8}$ Neste contexto, o questionário de frequência alimentar (QFA) tem sido considerado uma referência nos estudos epidemiológicos ao que se refere a avaliação do consumo alimentar ${ }^{9} \mathrm{e}$, especialmente, à ingestão nutricional, nomeadamente para avaliar a relação causal entre a alimentação e a ocorrência de desfechos clínicos como o aparecimento de doenças crônicas não transmissíveis. ${ }^{10}$ 
O presente estudo teve como objetivo verificar se existem diferenças importantes em alguns aspectos nutricionais entre dois grupos de gestantes acompanhadas em serviços de atenção pré-natal, na região metropolitana e em uma pequena cidade do interior do Estado de Minas Gerais, Brasil.

\section{Métodos}

O presente trabalho teve a participação de 240 gestantes, em idade entre 18 a 40 anos, em atendimento pré-natal no Ambulatório Jenny Faria, em Belo Horizonte (BH) e Posto de Saúde Padre Antônio Mendes, situado na cidade de Paula Cândido (PC), MG. As gestantes foram divididas em grupos, de acordo com cada trimestre, sendo 30 gestantes por trimestre na cidade do interior e 50 por trimestre na região metropolitana, totalizando 90 gestantes acompanhadas em Paula Cândido e 150 em Belo Horizonte. O quantitativo amostral foi baseado a partir da metodologia de diversos estudos publicados. 0 trabalho apresentou modelo do tipo transversal, havendo um contato com cada gestante.

Foi aplicado um questionário de frequência alimentar(QFA) contendo informações como idade, cor, escolaridade, estado civil, trabalho fora de casa e renda mensal. Além disto, foram aferidos o peso e estatura atual, assim como questionado o peso habitual anterior à gestação (peso pré-gestacional). Caso a gestante não se lembrasse, foi utilizado o primeiro peso no cartão da gestante, posteriormente foi calculado o IMC (índice de massa corpórea) pré-gestacional e atual.

Em relação à alimentação foram analisadas questões como: quantas refeições fazem ao dia, se tem costume de tomar café (com açúcar) toda hora, óleo utilizado nas refeições e consumo de refrigerante.

O QFA foi dividido em grupos alimentares e considerando os hábitos regionais. Utilizou-se como referência o questionário específico para gestantes, validado por Giacomello et al. ${ }^{11}$ Não foi utilizado lista de alimentos, como proposto pelo autor. Já que este é um trabalho qualitativo e visa conhecer a alimentação habitual das gestantes de acordo com a pirâmide alimentar. O QFA foi dividido nas seguintes frequências de consumo: todos os dias, 5 a 6 dias por semana, 3 a 4 dias por semana, 1 a 2 dias por semana, 1 a 3 vezes por mês e raramente/nunca. Foram incluídas a frequência de quantas vezes almoça ou janta fora de casa; troca almoço e jantar por lanche; consome frutas; consome salada de vegetais crus; salada de vegetais cozidos (exceto batata, mandioca, inhame); consome carne; consome frituras; se utiliza gordura de porco nas refeições e qual frequência; consumo de leite; consumo de café; consumo de pão, biscoito ou bolo; produtos de padaria integral; arroz, macarrão; angu; fastfood; alimentos ricos em açúcares e/ou gorduras; refrigerante e doces.

Os critérios de exclusão para o estudo foi a presença de diabetes ou outras doenças endócrinas, eclâmpsia ou préeclâmpsia, anemia ou outra deficiência nutricional previamente detectada em exame, além de vegetarianismo e intolerância a glúten e/ou lactose, pois poderia influenciar no hábito alimentar.

A ingestão alimentar habitual foi avaliada levando em consideração as porções recomendadas para cada grupo de alimentos na Pirâmide Alimentar Adaptada para gestantes ${ }^{2}$ e foi utilizado o parâmetro recomendado pelo Instituto de Medicina ${ }^{12}$ para ganho de peso em gestantes, de acordo com o trimestre.

Em relação à análise estatística, foi realizado uma análise descritiva para as variáveis quantitativas e qualitativas, com o uso de medidas de tendência central (usou-se o teste t para comparação de médias, uma vez que as suposições de normalidade foram garantidas).

Em um segundo momento, foi realizado uma comparação entre região metropolitana e interior, relacionando o ganho de peso com as variáveis de alimentação. A comparação foi feita de forma inferencial, utilizando o teste qui-quadrado de Independência para avaliar relação do ganho de peso com a alimentação, diferenciando para cada região. O nível de significância para todos os testes foi de $5 \%$, e utilizado o programa SPSS versão 15.0 .

\section{Resultados}

Em relação às variáveis socioeconômicas, em ambos os grupos, houve predomínio das gestantes que são casadas ( $\mathrm{BH}=56,6 \% ; \mathrm{PC}=46,6 \%)$ e não trabalham fora de casa $(\mathrm{BH}=54,6 \%$; $\mathrm{PC}=84,4 \%)$, a variável escolaridade apresentou maior porcentagem de ensino médio completo na região metropolitana (46\%) e ensino fundamental incompleto na região interior $(28,8 \%)$ (-Fig. $\mathbf{1})$.

A comparação das médias entre interior e região metropolitana foi significante no trimestre 2 para as variáveis idade $(p=0,002)$, peso pré-gestacional $(p=0,005)$, peso atual $(p=0,0106)$ IMC pré-gestacional $(p=0,02)$ e IMC atual $(p=0,04)$. No $3^{\circ}$ trimestre houve significância apenas para variável peso atual $(p=0,03)$ ( - Tabela 1 ).

A frequência das refeições diárias obtiveram predomínio de 3 a 4 refeições por dia no $1^{\circ}$ e $2^{\circ}$ trimestres $(\mathrm{BH}=54,0$ e $46 \%$, respectivamente; $\mathrm{PC}=66,7$ e $63,3 \%$, respectivamente), exceto entre as gestantes do $3^{\circ}$ trimestre de $\mathrm{BH}$, que consumiam 5 a 6 refeições ao dia $(44 \% ; n=22)$. 0 ganho de peso foi dividido por trimestre e calculado o ganho ponderal significativo ou não, baseado nas recomendações propostas pelo IOM. ${ }^{12}$ Portanto, foram classificadas como "não houve ganho de peso" as gestantes que estavam dentro da faixa aceitável a partir do seu estado nutricional pré-gestacional. Sendo assim, de acordo com os resultados, houve ganho de peso somente no primeiro trimestre, nas regiões metropolitana e interior (58,0 e 53,3\%, respectivamente) (-Tabela 2 ).

Posteriormente, comparou se o ganho de peso é influenciável ou não por diversos hábitos alimentares. Houve diferença para as variáveis "almoça ou janta fora de casa," no $1^{\circ}$ trimestre para as gestantes de $\mathrm{BH}(p=0,006)$; "quantas vezes consome leite," no $1^{\circ}$ trimestre do grupo do interior $(p=0,03)$; "quantas vezes consome fastfood," no $3^{\circ}$ trimestre das gestantes de $\mathrm{BH}(p=0,009)$ ( - Tabela 3 ).*

\footnotetext{
A tabela completa, com todas as informações e valores de significância podem ser vistas em material suplementar (rbgo. com.br).
} 


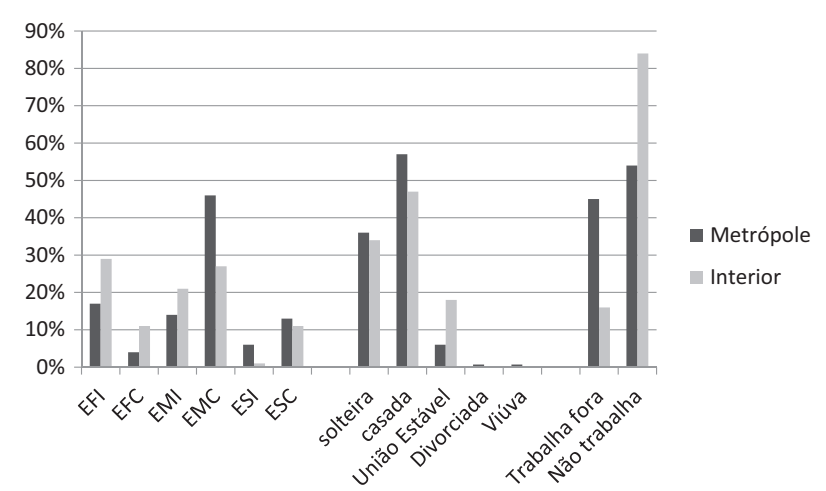

Fig. 1 Características socioeconômicas das gestantes entrevistadas, nas regiões metropolitana e interior.

Abreviações: $\mathrm{EFI}$, ensino fundamental incompleto; $\mathrm{EFC}$, ensino fundamental completo; EMI, ensino médio incompleto; EMC, ensino médio completo; ESI, ensino superior incompleto; ESC, ensino superior completo.

\section{Discussão}

Um resultado importante encontrado no estudo foi a prevalência de sobrepeso no período pré-gestacional na região metropolitana, já que todas as médias de IMC se enquadraram na faixa de sobrepeso. Além disto, foi observado ganho de peso significativo somente no primeiro trimestre em ambas as regiões, no segundo e terceiro trimestres a maioria das gestantes obtiveram ganho de peso dentro do recomendado.

A gestação pode atuar como desencadeante da obesidade ou como agravante, quando esta for preexistente. Na avaliação do estado nutricional durante período da gestação, houve um aumento no sobrepeso quando comparados com o período pré-gestacional, semelhantes a outros estudos. ${ }^{13} \mathrm{Em}$ relação ao período gestacional, o maior risco para complicações relacionadas ao ganho de peso inadequado são para as gestantes obesas, que estão suscetíveis a diabetes, hipertensão, parto cirúrgico, enquanto seus filhos são mais propensos a apresentarem microssomia, riscos de malformação fetal e maior mortalidade perinatal. ${ }^{14}$

O estado nutricional pré-gestacional é um dos principais fatores associados ao ganho de peso durante a gravidez. ${ }^{15} \mathrm{~A}$ chance elevada de ganho excessivo de peso em gestantes com sobrepeso e obesidade pré-gestacional confirma os achados de duas coortes, uma com 141 gestantes saudáveis de um serviço público do município de São Paulo ${ }^{16}$ e outra com 667 gestantes acompanhadas até o parto na rede básica de saúde do Estado do Rio Grande do Sul. ${ }^{17}$

Em relação às variáveis socioeconômicas, notamos que o nível de escolaridade é menor no interior e, além disto,

Tabela 1 Comparação das médias, por região, das variáveis idade, peso pré gestacional, peso atual, altura, IMC pré gestacional e IMC atual

\begin{tabular}{|c|c|c|c|c|c|c|c|c|}
\hline \multirow[t]{3}{*}{ Trimestres } & \multirow[t]{3}{*}{ Variáveis } & \multicolumn{6}{|c|}{ Região } & \multirow[t]{3}{*}{ Valor $p$} \\
\hline & & \multicolumn{3}{|c|}{ Metropolitana } & \multicolumn{3}{|c|}{ Interior } & \\
\hline & & $\mathrm{N}$ & Média & Desvio padrão & $\mathrm{N}$ & Média & Desvio padrão & \\
\hline \multirow[t]{6}{*}{ Trimestre 1} & Idade & 50 & 29,0800 & 6,9776 & 30 & 27,1333 & 6,8619 & 0,2278 \\
\hline & PPG & 50 & 66,7100 & 17,2069 & 30 & 65,8448 & 15,2434 & 0,8210 \\
\hline & PA & 50 & 69,6410 & 19,2132 & 30 & 68,0500 & 16,5144 & 0,7069 \\
\hline & Altura & 50 & 1,6180 & 0,0777 & 30 & 1,6097 & 0,0544 & 0,5755 \\
\hline & IMCPG & 50 & 27,6212 & 18,2511 & 30 & 25,7757 & 5,5977 & 0,5923 \\
\hline & IMCA & 50 & 28,6178 & 17,6039 & 30 & 26,2210 & 5,8661 & 0,4734 \\
\hline \multirow[t]{6}{*}{ Trimestre 2} & Idade & 50 & 28,0800 & 5,7812 & 30 & 24,2000 & 4,2051 & 0,0020 \\
\hline & PPG & 50 & 65,8160 & 18,6135 & 30 & 56,9700 & 8,5317 & $0,0050^{*}$ \\
\hline & PA & 50 & 70,7380 & 19,2241 & 30 & 62,2983 & 9,4756 & $0,0106^{*}$ \\
\hline & Altura & 50 & 1,6120 & 0,0745 & 30 & 1,5830 & 0,0684 & 0,0862 \\
\hline & IMCPG & 50 & 25,2588 & 6,7911 & 30 & 22,7197 & 2,9428 & $0,0239^{*}$ \\
\hline & IMCA & 50 & 27,1560 & 6,9664 & 30 & 24,8427 & 3,2956 & $0,0487^{*}$ \\
\hline \multirow[t]{6}{*}{ Trimestre 3} & Idade & 50 & 27,7600 & 6,9063 & 30 & 26,2069 & 7,1733 & 0,3401 \\
\hline & PPG & 50 & 67,0320 & 14,1164 & 30 & 61,7733 & 13,5451 & 0,1056 \\
\hline & PA & 50 & 77,7320 & 13,0585 & 30 & 71,2667 & 13,5195 & $0,0376^{*}$ \\
\hline & Altura & 50 & 1,6184 & 0,0604 & 30 & 1,5953 & 0,0604 & 0,1023 \\
\hline & IMCPG & 50 & 25,6312 & 5,4437 & 30 & 24,4700 & 6,2182 & 0,3840 \\
\hline & IMCA & 50 & 29,7092 & 4,9372 & 30 & 28,1977 & 6,3400 & 0,2377 \\
\hline
\end{tabular}

Abreviações: IMCPG, índice de massa corporal pré-gestacional; IMCA, índice de massa corporal atual; PA, peso atual; PPG, peso pré-gestacional. Teste $\mathrm{x}^{2}$ : associação significativa para* $\mathrm{p}<0,05$. 
Tabela 2 Número de refeições realizadas por dia e ganho de peso* acima do recomendado, por trimestre gestacional e região

\begin{tabular}{|c|c|c|c|c|c|c|c|}
\hline \multicolumn{3}{|c|}{ Trimestre Gestacional } & \multicolumn{2}{|c|}{$\begin{array}{l}\text { Região } \\
\text { Metropolitana }\end{array}$} & \multirow{2}{*}{$\begin{array}{l}\text { Interior } \\
\mathbf{n}\end{array}$} & & \multirow[t]{2}{*}{ Total } \\
\hline & & & $n$ & $\mathrm{Fr}(\%)$ & & $\operatorname{Fr}(\%)$ & \\
\hline \multirow[t]{8}{*}{ Trimestre 1} & \multirow[t]{4}{*}{ Faixa do $n^{\circ}$ de refeições } & 1 a 2 refeições & 2 & 4 & 3 & 10 & 5 \\
\hline & & 3 a 4 refeições & 27 & 54 & 20 & 66,7 & 47 \\
\hline & & 5 a 6 refeições & 14 & 28 & 7 & 23,3 & 21 \\
\hline & & $>6$ refeições & 7 & 14 & 0 & 0 & 7 \\
\hline & \multicolumn{2}{|l|}{ Total } & 50 & 100 & 30 & 100 & 80 \\
\hline & \multirow[t]{2}{*}{ Ganho de peso } & Não houve ganho & 21 & 42 & 14 & 46,7 & 35 \\
\hline & & Houve ganho & 29 & 58 & 16 & 53,3 & 45 \\
\hline & \multicolumn{2}{|l|}{ Total } & 50 & 100 & 30 & 100 & 80 \\
\hline \multirow[t]{8}{*}{ Trimestre 2} & \multirow[t]{4}{*}{ Faixa do $n^{\circ}$ de refeições } & 1 a 2 refeições & 1 & 2 & 4 & 13,33 & 5 \\
\hline & & 3 a 4 refeições & 23 & 46 & 19 & 63,33 & 42 \\
\hline & & 5 a 6 refeições & 20 & 40 & 7 & 23,33 & 27 \\
\hline & & $>6$ refeições & 6 & 12 & 0 & 0 & 6 \\
\hline & \multicolumn{2}{|l|}{ Total } & 50 & 100 & 30 & 100 & 80 \\
\hline & \multirow[t]{2}{*}{ Ganho de peso } & Não houve ganho & 38 & 76 & 24 & 80 & 62 \\
\hline & & Houve ganho & 12 & 24 & 6 & 20 & 18 \\
\hline & \multicolumn{2}{|l|}{ Total } & 50 & 100 & 30 & 100 & 80 \\
\hline \multirow[t]{8}{*}{ Trimestre 3} & \multirow[t]{4}{*}{ Faixa do $n^{\circ}$ de refeições } & 1 a 2 refeições & 2 & 4 & 5 & 16,7 & 7 \\
\hline & & 3 a 4 refeições & 17 & 34 & 16 & 53,3 & 33 \\
\hline & & 5 a 6 refeições & 22 & 44 & 8 & 26,7 & 30 \\
\hline & & $>6$ refeições & 9 & 18 & 1 & 3,3 & 10 \\
\hline & \multicolumn{2}{|l|}{ Total } & 50 & 100 & 30 & 100 & 80 \\
\hline & \multirow[t]{2}{*}{ Ganho de peso } & Não houve ganho & 35 & 70 & 24 & 80 & 59 \\
\hline & & Houve ganho & 15 & 30 & 6 & 20 & 21 \\
\hline & \multicolumn{2}{|l|}{ Total } & 50 & 100 & 30 & 100 & 80 \\
\hline
\end{tabular}

*O cálculo do ganho de peso foi realizado a partir das recomendações do Instituto de Medicina (1990), de acordo com o peso pré gestacional. IMC pré gestacional $(<19,8)$ : baixo peso. Ganho de peso de $2,3 \mathrm{~kg}$ no $1^{\circ}$ trimestre e ganho total entre $12,5-18 \mathrm{~kg}$; IMC pré gestacional $(19,8-26,0)$ : adequado. Ganho de peso de $1,6 \mathrm{~kg}$ no $1^{\circ}$ trimestre e ganho total entre $11,5-16 \mathrm{~kg}$; IMC pré gestacional $(26,0-29,0)$ : sobrepeso. Ganho de peso de $0,9 \mathrm{~kg}$ no $1^{\circ}$ trimestre e ganho total entre 7-11,5kg; IMC pré gestacional (>29,0): obesidade. Manter o peso pré gestacional $1^{\circ}$ trimestre e ganho total de $7 \mathrm{~kg}$.

menos gestantes se encontram inseridas no mercado de trabalho. A literatura refere que condições socioeconômicas desfavoráveis produzem resultados insatisfatórios na saúde da população em geral, e quanto maior a renda, maior o poder de compra e acesso a alimentação variada, ${ }^{18}$ o que pode influenciar na saúde materna e fetal. A baixa escolaridade pode ser vista como agravante na saúde das mulheres, pois é considerado pelo Ministério da Saúde como fator de risco obstétrico. ${ }^{19}$ Apesar da maioria das gestantes entrevistadas não ter alta escolaridade no interior, houve busca pelo serviço de saúde para do pré-natal, o que pode revelar uma tendência ao interesse pela sua saúde e de seus filhos.

Como dito anteriormente, foi observado nas gestantes de BH uma tendência a sobrepeso e maior nível de escolaridade, tal como em estudo realizado em São Paulo, em que gestantes com quatro anos ou mais de estudo apresentaram ganho de quase dois quilos a mais quando comparadas às demais, sendo a escolaridade considerada um marcador de acesso aos alimentos. ${ }^{6}$ Por outro lado, em Recife, a baixa escolaridade contribuiu para o ganho de peso excessivo, o nível de escolaridade reflete a situação socioeconômica, sendo assim, as gestantes com menor poder aquisitivo priorizam o consumo de alimentos mais calóricos (ricos em gorduras, particularmente de origem animal, açúcar e alimentos refinados, em detrimento aos carboidratos complexos e fibras) por terem menor custo. ${ }^{20}$

O número de refeições diárias é menor que o preconizado por Accioly et al, ${ }^{21}$ que recomenda 5 a 6 refeições por dia. A dieta deve ser fracionada com menores porções (café da manhã, lanche - opcional, almoço, merenda, jantar, ceia opcional com intervalo de $3 / 3 \mathrm{~h}$ ) para que ao longo do dia, haja aporte de nutrientes e energia necessários. No entanto, o estudo demonstrou predominância de 3 a 4 refeições por dia, estando adequado somente na região metropolitana no terceiro trimestre. A análise do consumo alimentar não demonstrou grande discrepância com o recomendado pela 


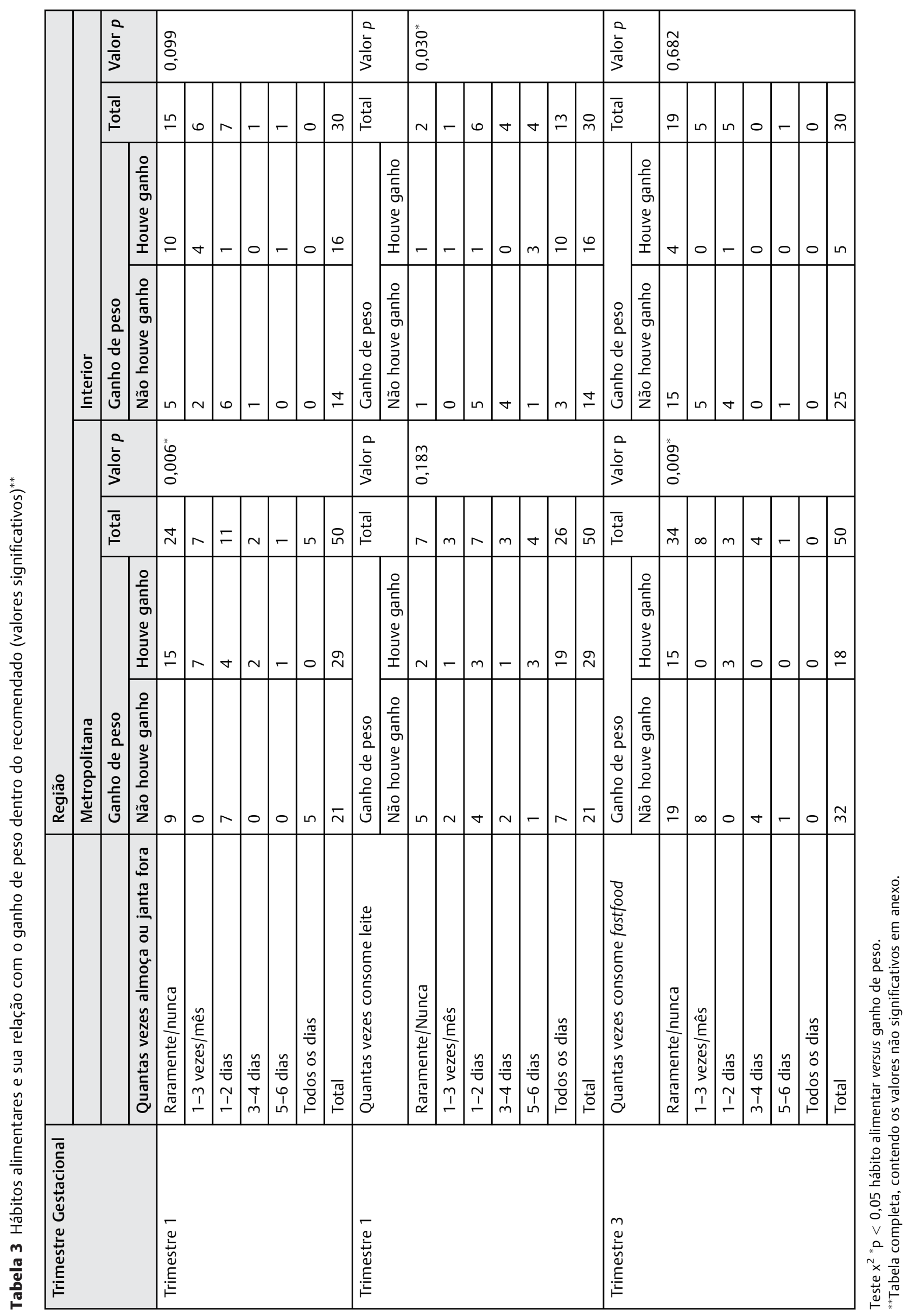


pirâmide alimentar. Houve prevalência no consumo diário de frutas, verduras e vegetais, leguminosas, cereais, leite e carnes e baixo consumo de fastfood, alimentos ricos em açúcares e/ou gorduras e refrigerante. Isto pode ser consequência do fato de uma nutricionista aplicar os questionários, levando as gestantes a informar uma alimentação saudável, já que as mesmas possuem informações sobre alimentação saudável, porém, nem sempre as colocam em prática. $^{22}$

Uma limitação do estudo foi a utilização do QFA como método de avaliação do consumo alimentar, uma vez que ele contém grupos de alimentos, limitando uma comparação com história dietética. 23

Estudos recentes demonstram que alimentos processados e industrializados tem sido identificado entre as gestantes em diversas regiões do mundo e reflete as mudanças ocorridas no mundo moderno, como a busca por maior comodidade, praticidade e rapidez, que afetam diretamente a alimentação da população. ${ }^{24-27}$ No Brasil, similarmente às demais regiões do mundo, essas modificações estão relacionadas com alterações no estilo de vida, que se expressam pela redução do gasto calórico diário e na adoção de hábitos alimentares caracterizados por elevado consumo de gorduras saturadas, de açúcares simples, produtos industrializados e redução no consumo de frutas, verduras e legumes. Este padrão de consumo alimentar comprova a tendência de modificação dos hábitos alimentares registrados por pesquisas ao longo do tempo. ${ }^{28}$

Nota-se prevalência de consumo de frutas, leite e salada de vegetais crus ou cozidos (folhas, leguminosas, raízes, etc). Estes hábitos contribuem com a adequação de proteínas e carboidratos simples e complexos e dos micronutrientes (vitaminas e minerais). Assim, podem estar associados a melhores condições de saúde da gestante e ao crescimento e desenvolvimento fetal. Deve-se salientar que esses padrões apresentam em suas composições nutrientes essenciais para a saúde humana e são considerados os mais próximos das recomendações dietéticas emanadas atualmente para gestação e como tal, pode ser o mais intuitivamente associado às melhores condições de saúde na gestação, ao crescimento e desenvolvimento adequado do feto. ${ }^{29}$ Em estudo realizado por Belarmino et $\mathrm{al}^{30}$ foi investigado o consumo alimentar de acordo com os grupos de alimentos que compõem a pirâmide alimentar, sendo categorizados nos seis grupos de alimentos: grupo A (pão, arroz, cereais e massas); grupo B (vegetais e frutas); grupo C (carnes, aves, peixes e ovos); grupo $\mathrm{D}$ (feijão e nozes); grupo E (leite, iogurte e queijo); e grupo $\mathrm{F}$ (gorduras, óleos e doces). No trabalho supracitado, verificou-se o baixo consumo de alimentos do grupo $\mathrm{B}$, diferente do que foi encontrado no presente estudo.

Muito ainda há que ser feito para que mais gestantes atentem para educação nutricional satisfatória. Só assim, elas poderão compreender melhor o período e as mudanças que estão vivenciando e poderão praticar hábitos alimentares saudáveis.

0 presente estudo permitiu concluir que as gestantes da região metropolitana e interior, de modo geral, se alimentam de maneira adequada, com hábitos alimentares diversifica- dos e favoráveis à manutenção da saúde, rico em frutas e verduras. Porém, também se verificou um perfil sociodemográfico pouco favorável ao período gestacional, com baixa escolaridade e renda das entrevistadas, principalmente no interior. Sugere-se que estudos mais detalhados sejam realizados, verificando a associação entre o perfil alimentar da gestante e a saúde do recém-nascido, para que seja possível identificar os fatores de risco gestacionais para a saúde da mãe e do concepto.

Agradecimentos

À Coordenação de Aperfeiçoamento de Pessoal de Nível Superior - CAPES, pelo auxílio recebido (bolsa de mestrado).

\section{Referências}

1 U.S. Department of Health and Human Services. U.S. Department of Agriculture [Internet]. Dietary guidelines for Americans, 2005. Washington (DC): U.S. Government Printing Office; 2005 [cited 2015 Mar 12]. Available from: <http://health.gov/dietaryguidelines/dga2005/document/pdf/DGA2005.pdf >

2 Demetrio F. Pirâmide alimentar para gestantes eutróficas de 19 a 30 anos. Rev Nutr 2010;23(5):763-778

3 Kowal C, Kuk J, Tamim H. Characteristics of weight gain in pregnancy among Canadian women. Matern Child Health J 2012;16(3):668-676

4 Padilha PC, Saunders C, Machado RCM, et al. [Association between pre-gestational nutritional status and prediction of the risk of adverse pregnancy outcome]. Rev Bras Ginecol Obstet 2007;29(10):511-518

5 Nomura RMY, Paiva LV, Costa VN, Liao AW, Zugaib M. [Influence of maternal nutritional status, weight gain and energy intake on fetal growth in high-risk pregnancies]. Rev Bras Ginecol Obstet 2012;34(3):107-112 Portuguese.

6 Konno SC, Benicio D’ Aquino MH, Barros AJD. Fatores associados à evolução ponderal de gestantes: uma análise multinível. Rev Saude Publica 2007;41(6):995-1002

7 Phelan S, Phipps MG, Abrams B, Darroch F, Schaffner A, Wing RR. Randomized trial of a behavioral intervention to prevent excessive gestational weight gain: the Fit for Delivery Study. Am J Clin Nutr 2011;93(4):772-779

8 Cade J, Thompson R, Burley V, Warm D. Development, validation and utilisation of food-frequency questionnaires - a review. Public Health Nutr 2002;5(4):567-587

9 Pinto E, Severo M, Correia S, dos Santos Silva I, Lopes C, Barros H. Validity and reproducibility of a semi-quantitative food frequency questionnaire for use among Portuguese pregnant women. Matern Child Nutr 2010;6(2):105-119

10 Slater B, Philippi ST, Marchioni DML, Fisberg RM. Validação de Questionários de Frequência Alimentar - QFA: considerações metodológicas. Rev Bras Epidemiol 2003;6(3):200-208

11 Giacomello A, Schmidt MI, Nunes MAA, et al. Validação relativa de Questionário de Frequência Alimentar em gestantes usuárias de serviços do Sistema Único de Saúde em dois municípios no Rio Grande do Sul, Brasil. Rev Bras Saude Mater Infant 2008;8(4): 445-454

12 National Academy of Science. Institute of Medicine. Nutrition during pregnancy: Part I: weight gain, Part II: nutrient supplements. Washington (DC): National Academies Press; 1990

13 Parizzi MR, Fonseca JGM. Nutrição na gravidez e na lactação. Rev Med Minas Gerais 2010;20(3):341-353 
14 Gonçalves CV, Mendoza-Sassi RA, Cesar JA, de Castro NB, Bortolomedi AP. [Body mass index and gestational weight gain as factors predicting complications and pregnancy outcome]. Rev Bras Ginecol Obstet 2012;34(7):304-309

15 Gunderson EP, Murtaugh MA, Lewis CE, Quesenberry CP, West DS, Sidney S. Excess gains in weight and waist circumference associated with childbearing: The Coronary Artery Risk Development in Young Adults Study (CARDIA). Int J Obes Relat Metab Disord 2004;28(4):525-535

16 Stulbach TE, Benício MHD, Andreazza R, Kono S. Determinantes do ganho ponderal excessivo durante a gestação em serviço público de pré-natal de baixo risco. Rev Bras Epidemiol 2007; 10(1):99-108

17 Drehmer M, Camey S, Schmidt MI, et al. Socioeconomic, demographic and nutritional factors associated with maternal weight gain in general practices in Southern Brazil. Cad Saude Publica 2010;26(5):1024-1034

18 Monteiro CA, Mondini L, Costa RBL. Mudanças na composição e adequação nutricional da dieta familiar nas áreas metropolitanas do Brasil entre 1988 e 1996. In: Monteiro CA, organizador. Velhos e novos males da saúde no Brasil: a evolução do país e de suas doenças. 2a ed. São Paulo: Hucitec; 2000:359-74

19 Brasil. Ministério da saúde. Secretaria de Políticas Públicas. Departamento de Gestão de Políticas Estratégicas. Área Técnica de Saúde da Mulher. Gestação de alto risco: manual técnico. 3a ed. Brasília (DF): Ministério da Saúde; 2000

20 Andreto LM, de Souza AI, Figueiroa JN, Cabral-Filho JE. Fatores associados ao ganho ponderal excessivo em gestantes atendidas em um serviço público de pré-natal na cidade de Recife, Pernambuco, Brasil. Cad Saude Publica 2006;22(11):2401-2409

21 Accioly E, Saunders C, Lacerda EMA. Nutrição em obstetrícia e pediatria. 2a ed. Rio de Janeiro: Cultura Médica/Guanabara Koogan; 2010
22 Reticena KO, Mendonça FF. Perfil alimentar de gestantes atendidas em um hospital da região noroeste do Paraná. UNOPAR Cient Ciênc Biol Saúde 2012;14(2):99-104

23 Waijers PM, Feskens EJ, Ocké MC. A critical review of predefined diet quality scores. Br J Nutr 2007;97(2):219-231

24 Brasil. Ministério da Saúde. Secretaria de Atenção à Saúde. Coordenação-Geral da Política de Alimentação e Nutrição. Guia alimentar para a população brasileira: promovendo a alimentação saudável. Brasília (DF): Ministério da Saúde; 2005

25 Lange NE, Rifas-Shiman SL, Camargo CA Jr, Gold DR, Gillman MW, Litonjua AA. Maternal dietary pattern during pregnancy is not associated with recurrent wheeze in children. J Allergy Clin Immunol 2010;126(2):250-255, 255.e1-255.e4

26 Musselman JR, Jurek AM, Johnson KJ, et al. Maternal dietary patterns during early pregnancy and the odds of childhood germ cell tumors: A Children's Oncology Group study. Am J Epidemiol 2011;173(3):282-291

27 McGowan CA, McAuliffe FM. Maternal dietary patterns and associated nutrient intakes during each trimester of pregnancy. Public Health Nutr 2013;16(1):97-107

28 Instituto Brasileiro de Geografia e Estatística [Internet]. Pesquisa de orçamentos familiares 2008-2009: antropometria de crianças, adolescentes e adultos no Brasil. Rio de Janeiro: IBGE; 2010 [cited 2015 Apr 2]. Available at: http://www.ibge.gov.br/home/estatistica/populacao/condicaodevida/pof/2008_2009_encaa/pof_20082009_encaa.pdf

29 Brasil. Ministério da Saúde. Secretária de Atenção a Saúde. Departamento de Atenção Básica. Guia alimentar da população brasileira: promovendo a alimentação saudável. Brasília (DF): Ministério da Saúde; 2006

30 Belarmino GO, Moura ERF, Oliveira NC, Freitas GL. Risco nutricional entre gestantes adolescentes. Acta Paul Enferm 2009; 22(2):169-175 